\title{
TWO NEW RECORDED SPECIES OF THE FAMILY LEPTONCHIDAE (NEMATODA: DORYLAIMIDA) FOR VIETNAM'S FAUNA FROM CUC PHUONG NATIONAL PARK
}

\author{
Nguyen Thi Anh Duong ${ }^{*}$, Vu Thi Thanh Tam ${ }^{1}$, Reyes Peña-Santiago ${ }^{2}$ \\ ${ }^{(1)}$ Institute of Ecology and Biological Resources, VAST, ${ }^{*}$ nad2807@yahoo.com \\ ${ }^{(2)}$ University of Jaén, Spain
}

\begin{abstract}
Proleptonchus aestivus and Tyleptus projectus from Cuc Phuong natural forest are described and illustrated for the first time in Vietnam. Proleptonchus aestivus species is characterized by its body 1.14-1.21 mm long; lip region cap-like with lips amalgamated and papillae little protruding; odontostyle very slender and odontophore rod-like, arcuate; female genital system mono-prodelphic; tail short and rounded, hemispheroid. Tyleptus projectus species is characterized by its body $0.79-0.95 \mathrm{~mm}$ long; lip region set off by marked depression or weak constriction; six lips conspicuous projecting liplets around the oral opening; odontostyle small but robust, odontophore rod-like; female genital system monoopisthodelphic, male genital system diorchic, with opposite testes. Tail short and rounded, to hemispheroid.
\end{abstract}

Keywords: Dorylaimida, Leptonchidae, Proleptonchus aestivus, Tyleptus projectus, morphology, Vietnam.

\section{INTRODUCTION}

Free-living terrestrial nematode Leptonchidae is a poorly known dorylaimid family in Vietnam, with only one described species Thornedia opisthodelphic Jairajpuri, 1968 [4], which was collected from Cuc Phuong National forest in northern Vietnam. In this paper, two species of Proleptonchus aestivus and Tyleptus projectus in this area are recorded for the first time in Vietnam. Based on high resolution microphotographs, our study allows having better characterization of those species.

\section{MATERIAL AND METHODS}

Soil samples were collected from a pristine tropical forest in Cuc Phuong National Park (Vietnam). Nematodes were extracted from soil sample by modified Baermann funnel technique, killed by heat, fixed in formaldehyde $4 \%$, transferred to anhydrous glycerol according to Siddiqi (1964) [12], and mounted on glass slides for their handling. Specimens were photographed with a Nikon Eclipse 80i microscope and a Nikon DS digital camera. Raw photographs were edited using Adobe ${ }^{\circledR}$ Photoshop ${ }^{\circledR}$ CS.

\section{DESCRIPTION}

\section{Proleptonchus aestivus Lordello, 1955 (Fig. 1)}

Material examined: Two females, in good condition.

\section{Measurements: See table 1.}

Female: Moderately slender nematodes of medium size, 1.14-1.21 mm long. Habitus after fixation slightly curved ventrad to an open $\mathrm{C}$ shape. Body cylindrical, tapering towards both ends, but more so anteriorly since the tail is rounded. Cuticle typical tylencholaimoid, with very irregular inner layer and provided with numerous dot-like radial elements. Lateral chord 10-14 $\mu \mathrm{m}$ wide or occupying one-fourth (26-28\%) of mid-body diameter, lacking any differentiation. Lip region cap-like, offset by marked depression or weak constriction, 2.1-2.3 times as wide as high, and about one-fifth (20$22 \%$ ) of body diameter at neck base; lips amalgamated; papillae little protruding.

Amphid fovea not observed. Stoma flaskshaped, distinctly sclerotized in its posterior half. Odontostyle very slender but with distinct lumen, slightly arcuate, approximately 10-12 times as long as wide, 0.6-0.8 times longer than lip region diameter or $0.44-0.50 \%$ of total body length. Odontophore rod-like, arcuate. Guiding ring simple, located at $7.0-8.0 \mu \mathrm{m}$ or one lip 
region diameter from anterior end. Pharynx consisting of a weakly muscular anterior portion and basal bulb, both parts separate by a distinct constriction; bulb 2.0-2.4 times as long as broad and as long as corresponding body diameter long, occupying about one-fifth (19-20\%) of total neck length. Pharyngeal gland nuclei obscure. Cardia short and rounded, about a quarter of body width. Genital system monoprodelphic; anterior branch well developed, 245 $\mu \mathrm{m}$ long, while the posterior one is reduced to an uterine sac $56 \mu \mathrm{m}$ long or 1.6 times the body diameter; anterior ovary large, $97 \mu \mathrm{m}$ long, with oocytes first in two or more rows, then in one row; oviduct $178 \mu \mathrm{m}$ or 4.7 body diameter long, consisting of a slender portion with prismatic cells and a very well developed pars dilatata with distinct lumen; sphincter well marked between oviduct and uterus; uterus a simple tube-like structure, $70 \mu \mathrm{m}$ long or 1.8 times of body diameter; vagina extending inwards $23 \mu \mathrm{m}$ or approximately one-half $(50 \%)$ of body diameter: pars proximalis $20 \times 18 \mu \mathrm{m}$, with convergent walls and enveloped by circular musculature in its distal portion, pars refringens absent, and pars distalis $6.0 \mu \mathrm{m}$ long; vulva a post-equatorial transverse slit. Prerectum 2.7 and rectum 1.4 anal body diameter long. Tail short and rounded, hemispheroid.

Male: Not found.

Table 1. Morphometric data of Proleptonchus aestivus. All measurements are in $\mu \mathrm{m}$ except ratios $\mathrm{a}, \mathrm{b}, \mathrm{c}, \mathrm{V}, \mathrm{c}^{\prime}$ and $\mathrm{L}$ in $\mathrm{mm}$

\begin{tabular}{|c|c|c|c|}
\hline \multirow{4}{*}{ Distribution } & \multicolumn{3}{|c|}{ Proleptonchus aestivus } \\
\hline & Brazil & India & Vietnam \\
\hline & Lordello (1955) & Jairajpuri (1964) & Present paper \\
\hline & Type material & Paratype & \\
\hline $\mathrm{n}$ & 10 우 & $50+9$ & 299 \\
\hline \multicolumn{4}{|l|}{ Character } \\
\hline $\mathrm{L}$ & $1.3-1.5$ & $1.2-1.5$ & $1.14-1.21$ \\
\hline $\mathrm{a}$ & $30-35$ & $32-35$ & $24-30$ \\
\hline $\mathrm{b}$ & $6.0-7.3$ & $6.0-7.5$ & $5.8-5.8$ \\
\hline $\mathrm{c}$ & $70-80$ & $70-75$ & $76-93$ \\
\hline $\mathrm{V}$ & $52-60$ & $52-58$ & $59-61$ \\
\hline$c^{\prime}$ & $?$ & $?$ & $0.6-0.8$ \\
\hline Lip region diameter & $?$ & $?$ & $7.5-8.0$ \\
\hline Odontostyle length & $?$ & $?$ & $5.0-6.0$ \\
\hline Odontophore length & $?$ & $?$ & $10.0-13.0$ \\
\hline Guiding ring from anterior end & $?$ & $?$ & $7.0-8.0$ \\
\hline Neck length & $?$ & $?$ & $195-207$ \\
\hline Pharyngeal expansion length & $?$ & $?$ & $40-40$ \\
\hline Body diameter - neck base & $?$ & $?$ & $37-41$ \\
\hline Body diameter - mid-body & $?$ & $?$ & $38-50$ \\
\hline Body diameter - anus & $?$ & $?$ & $20-22$ \\
\hline Prerectum length & $?$ & $?$ & $?-60$ \\
\hline Rectum length & $?$ & $?$ & $?-30$ \\
\hline Tail length & $?$ & $?$ & $13-15$ \\
\hline
\end{tabular}

?. no information. 

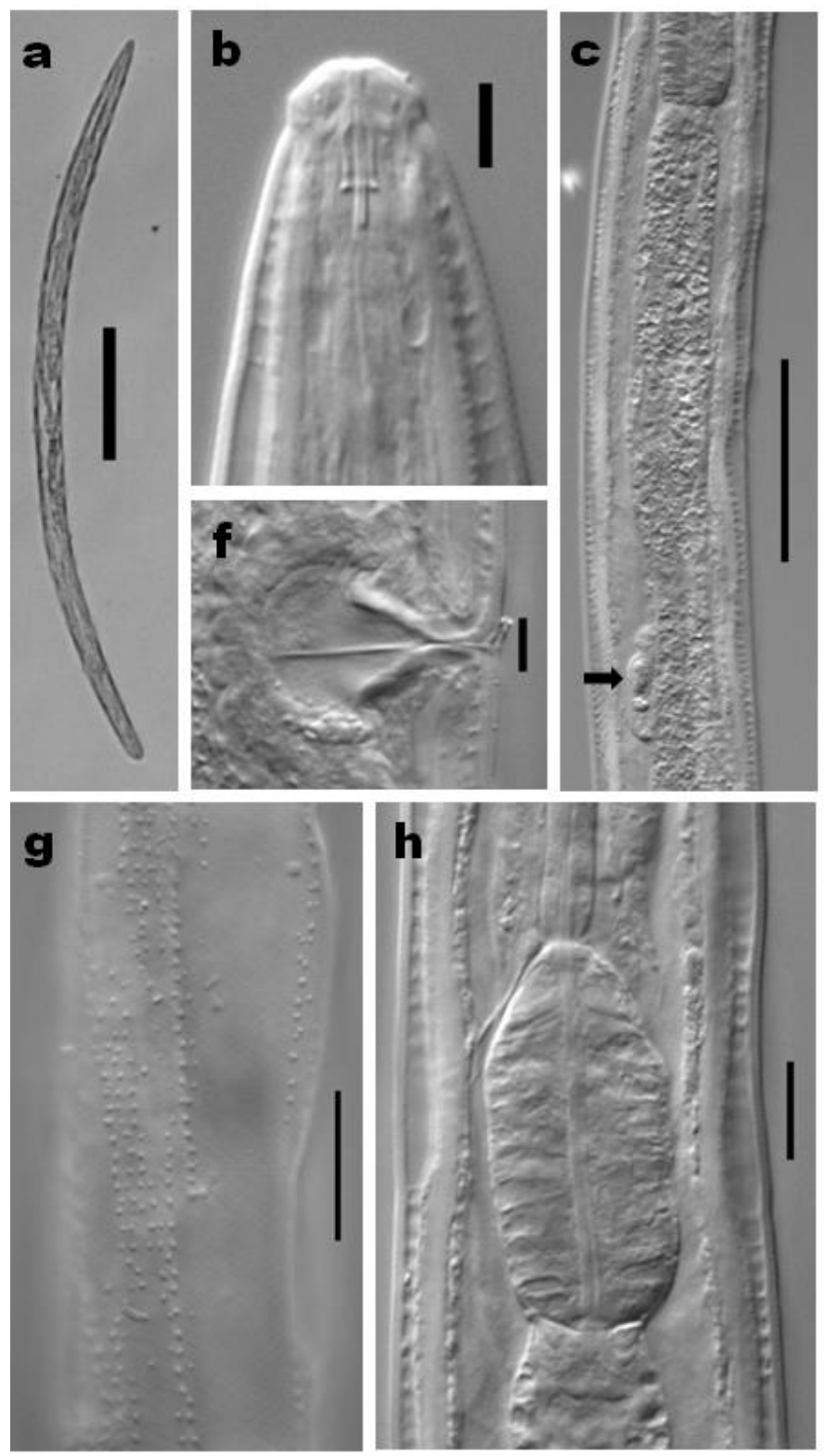

Figure 1. Proleptonchus aestivus Lordello, 1955 (female, LM)

a. Female, entire; b. Anterior region in median view; c. Body region behind pharyngeal bulb showing a pseudocelomocyte; $d$. Genital system; e. Neck region; f. Vagina; g. Cuticle in submedian view; h. Pharyngeal bulb and cardia; i. Caudal region (Scale bars: $\mathrm{a}=200 \mu \mathrm{m} ; \mathrm{b}, \mathrm{f}=5 \mu \mathrm{m}$; $\mathrm{c}-\mathrm{e}=50 \mu \mathrm{m} ; \mathrm{g}=20 \mu \mathrm{m} ; \mathrm{h}, \mathrm{i}=10 \mu \mathrm{m})$.

\section{Remarks}

The two Vietnamese specimens correspond well to type population from Brazil $[5,6,11]$ as well as Indian specimens reported by Jairajpuri
\& Siddiqi (1964) [8], with no significant difference observed.

Baqri (1991) [1] described an Indian population of $P$. clarus Timm $[6,9,14]$ which 
does not fit other populations of this species due to its smaller body in females (1.14-1.38, $\mathrm{n}=5 \mathrm{vs}$ 1.43-1.83 mm, $\mathrm{n}=10)$, shorter posterior uterine sac (51-68 $\mu \mathrm{m}$ or 1.1-1.7 body diameters $v s$ 98$129 \mu \mathrm{m}$ or $2.0-2.5$ body diameters) and male absent ( $v s$ as frequent as females). This population is not distinguishable from others belonging to $P$. aestivus, also known to occur in India.

Choi \& Jairajpuri (1998) [2] described the new species $P$. parkerus from Korea, very similar to $P$. aestivus, which was not compared to. These two species might be identical.

\section{Tyleptus projectus Thorne, 1939 (Fig. 2)}

Material examined: Seven females and one male, in very good condition.

Measurements: See table 2.

Adult: Moderately slender to slender nematodes of medium size, 0.79-0.95 mm long. Habitus after fixation curved ventrad to open Cshaped. Body cylindrical, tapering towards both ends, but more so at anterior body region. Cuticle typical tylencholaimoid, outer cuticle without visible transverse striation while the inner one shows irregular outline; radial refractive elements present. Lateral chord $7 \mu \mathrm{m}$ wide or occupying one-third of mid-body diameter, with two lines of coarse ducts reaching to their large pores. Lip region set off by marked depression or weak constriction, 1.8-2.0 times as wide as high and approximately one-half $(50 \%)$ of body diameter, at neck base; six lips conspicuous projecting liplets around the oral opening. Amphid fovea stirrupshaped, opening at level of cephalic depression, its aperture occupying $6.0 \mu \mathrm{m}$ or approximately three-fourths of lip region diameter. Cheilostom truncate cone, lacking any differentiation.

Odontostyle typical of the genus, small but robust, with distinct lumen and aperture that occupies one-fifth of total its length. Guiding ring simple, located at $5.0 \mu \mathrm{m}$ or 0.6 lip region diameter from anterior end. Odontophore rodlike, 1.3 times as long as odontostyle. Pharynx consisting of a slender, weakly muscular and very convoluted anterior portion and a pyriform basal bulb as long as the body diameter at neck base. Pharyngeal gland nuclei obscure. Cardia short and rounded. Prerectum 4.3, rectum 1.7 anal body diameter long. Tail short and rounded, to hemispheroid; its inner core imperfect irregular and sunken.

Female

Genital system mono-opisthodelphic. Anterior branch reduced to a short rudimentary pouch as long as body diameter. Posterior branch well developed, 215-220 $\mu \mathrm{m}$; reflex ovary, 60-70 $\mu \mathrm{m}$; oocytes first in two or more rows, then in one row. Oviduct $90 \mu \mathrm{m}$ long or 3.0-3.3 body diameter long and consisting of a slender portion with prismatic cells and a moderately developed pars dilatata with distinct lumen. Oviduct and uterus are separated by sphincter. Uterus a simple tube-like structure, $140 \mu \mathrm{m}$ long or 5.0 times the body diameter. Vagina extending inwards 13-14 $\mu \mathrm{m}$ : pars proximalis wider than long, with convergent walls and enveloped by weak circular musculature pars refringens absent, and pars distalis $3.0 \mu \mathrm{m}$ long. Vulva a preequatorial, transverse slit.

\section{Male}

Genital system diorchic, with opposite testes. In addition to the ad-cloacal pair, situated at $6.0 \mu \mathrm{m}$ from cloacal aperture, there are three ventromedian supplements, the posteriormost of which is situated out of the range of spicules and at $33 \mu \mathrm{m}$ from ad-cloacal pair. Spicules dorylaimoid, curved ventrad and relatively robust, 5.8 times as long as wide and 1.9 times as long as anal body diameter. Lateral guiding pieces $9.0 \mu \mathrm{m}$ long, 6.0 times as long as wide.

\section{Remarks}

The above description fits well that of type material $[6,13]$ and later records of this species [9, 10]. Nevertheless, T. projectus belongs to a group of very similar (if not identical) species, namely $T$. oryzae Dhanam \& Jairajpuri, 1999 [3], T. striatus Heyns, 1963 [7] and T. variabilis Jairajpuri \& Siddiqi, 1964 [8], which was never compared in depth and should be matter of a detailed study. 
Table 2. Morphometric data of Tyleptus projectus. All measurements are in $\mu \mathrm{m}$ except ratios a, b, c, $\mathrm{V}, \mathrm{c}^{\prime}$ and $\mathrm{L}$ in $\mathrm{mm}$

\begin{tabular}{|c|c|c|c|c|}
\hline \multirow{4}{*}{ Distribution } & \multicolumn{4}{|c|}{ Tyleptus projectus } \\
\hline & \multicolumn{2}{|r|}{ USA } & \multicolumn{2}{|c|}{ Vietnam } \\
\hline & Thorne (1939) & Goseco et al. (1974) & & t paper \\
\hline & Type material & Paratype & \multicolumn{2}{|c|}{ Paratype } \\
\hline $\mathrm{n}$ & 509 & 11 우우 & $10^{\pi}$ & 7 우우 \\
\hline $\mathrm{L}$ & $\begin{array}{l}1.18 \pm 0.10 \\
(1.07-1.28)\end{array}$ & $\begin{array}{l}1.07 \pm 0.08 \\
(0.90-1.17)\end{array}$ & 0.79 & $\begin{array}{l}0.90 \pm 0.03 \\
(0.87-0.95)\end{array}$ \\
\hline $\mathrm{a}$ & $\begin{array}{l}30.3 \pm 4.4 \\
(25.6-36.3) \\
\end{array}$ & $\begin{array}{l}28 \pm 1.8 \\
(25-31)\end{array}$ & 41.4 & $\begin{array}{c}35.7 \pm 2.2 \\
(33.0-37.9) \\
\end{array}$ \\
\hline $\mathrm{b}$ & $\begin{array}{l}5.1 \pm 0.2 \\
(4.8-5.2)\end{array}$ & $\begin{array}{c}4.5 \pm 0.4 \\
(3.8-5)\end{array}$ & 3.3 & $\begin{array}{l}3.9 \pm 0.3 \\
(3.6-4.5)\end{array}$ \\
\hline $\mathrm{c}$ & $\begin{array}{l}67.9 \pm 5.5 \\
(64-74.3)\end{array}$ & $\begin{array}{l}78.3 \pm 17.9 \\
(56-105)\end{array}$ & 56.2 & $\begin{array}{r}64.6 \pm 6.8 \\
(58.1-75.1)\end{array}$ \\
\hline V & $\begin{array}{l}33.4 \pm 1.4 \\
(32-35.3)\end{array}$ & $\begin{array}{l}33 \pm 2.3 \\
(31-37) \\
\end{array}$ & 0.71 & $\begin{array}{c}37 \pm 2 \\
(35-38)\end{array}$ \\
\hline c' & ? & ? & 0.9 & $\begin{array}{l}0.8 \pm 0.0 \\
(0.8-0.8)\end{array}$ \\
\hline Lip region diameter & ? & ? & 9.0 & $\begin{array}{c}8.8 \pm 0.8 \\
(8.0-10.0) \\
\end{array}$ \\
\hline Odontostyle length & ? & ? & 9.0 & $\begin{array}{l}9.0 \pm 0.0 \\
(9.0-9.0) \\
\end{array}$ \\
\hline Odontophore length & ? & ? & 10.0 & $\begin{array}{r}10.0 \pm 0.0 \\
(10.0-10.0)\end{array}$ \\
\hline $\begin{array}{l}\text { Guiding ring from anterior } \\
\text { end }\end{array}$ & ? & ? & 6.0 & $\begin{array}{l}5.2 \pm 0.4 \\
(5.0-6.0) \\
\end{array}$ \\
\hline Neck length & $\begin{array}{l}235 \pm 9.5 \\
(220-246)\end{array}$ & $\begin{array}{c}234.9 \pm 20.3 \\
(200-237)\end{array}$ & 235 & $\begin{array}{r}229 \pm 17.7 \\
(200-243) \\
\end{array}$ \\
\hline $\begin{array}{l}\text { Pharyngeal expansion } \\
\text { length }\end{array}$ & ? & ? & 28 & $\begin{array}{l}27.4 \pm 1.9 \\
(25.0-30.0) \\
\end{array}$ \\
\hline Body diameter - neck base & ? & ? & 19.0 & $\begin{array}{l}23.8 \pm 1.1 \\
(23.0-25.0)\end{array}$ \\
\hline Body diameter - mid-body & ? & ? & 19.0 & $\begin{array}{c}25.2 \pm 2.2 \\
(23.0-28.0)\end{array}$ \\
\hline Body diameter - anus & ? & ? & 15.0 & $\begin{array}{c}18.2 \pm 1.8 \\
(16.0-20.0) \\
\end{array}$ \\
\hline Prerectum length & $\begin{array}{c}66.4 \pm 17.2 \\
(46-84.4)\end{array}$ & $\begin{array}{l}69.3 \pm 20.6 \\
(48-106)\end{array}$ & 65 & ? \\
\hline Rectum length & ? & ? & 25 & $\begin{array}{c}14.0 \pm 1.4 \\
(12.0-15.0) \\
\end{array}$ \\
\hline Tail length & $\begin{array}{l}17.6 \pm 2.7 \\
(14.4-19.2)\end{array}$ & $\begin{array}{c}14.5 \pm 4.1 \\
(10-21)\end{array}$ & 14.0 & $\begin{array}{c}17.4 \pm 1.3 \\
(15.0-18.0)\end{array}$ \\
\hline Spicules length & - & - & 29 & - \\
\hline $\begin{array}{l}\text { Ventromedian } \\
\text { supplements }\end{array}$ & - & - & 3 & - \\
\hline
\end{tabular}

?. no information. 

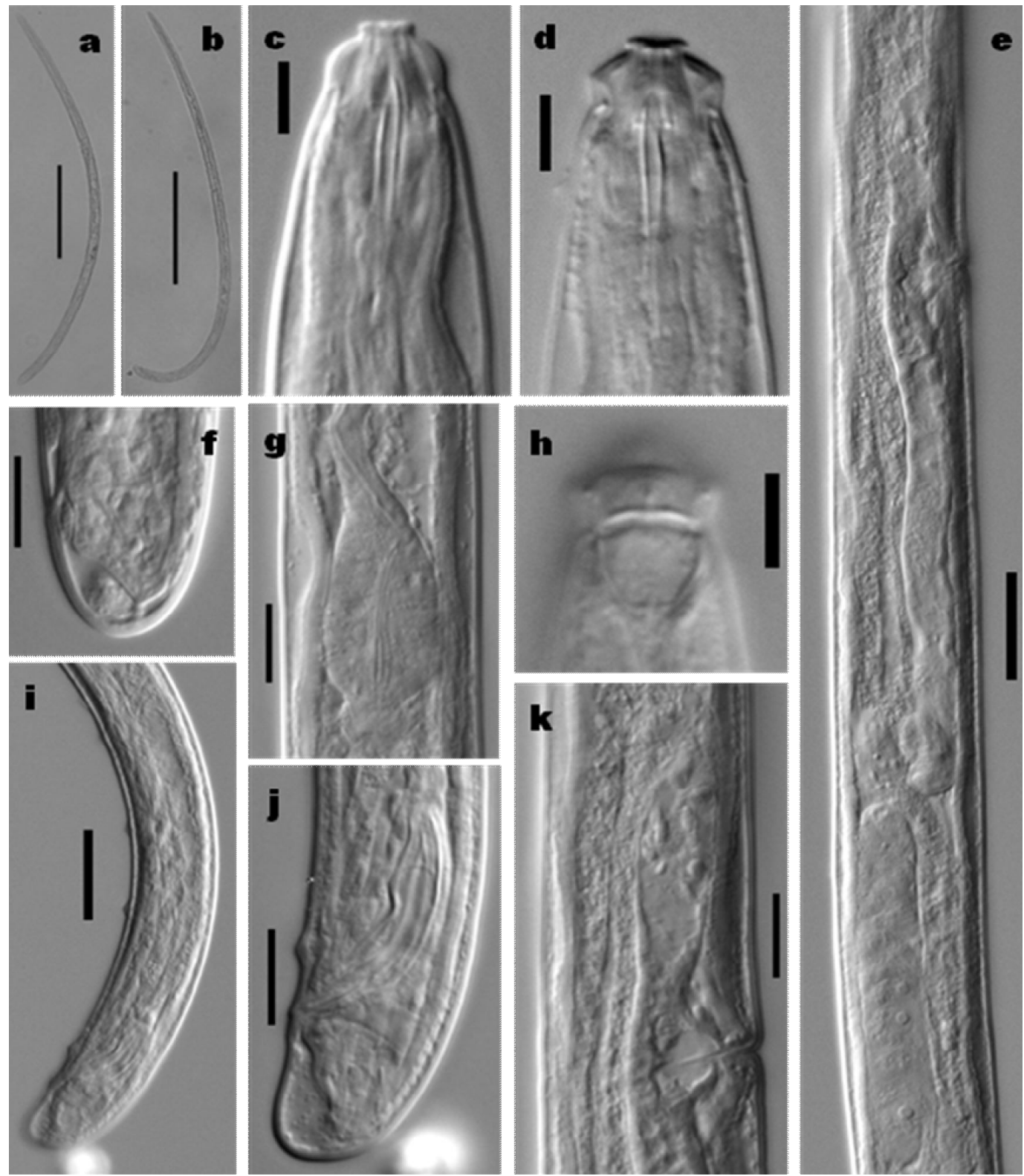

Figure 2. Tyleptus projectus Thorne, 1939 (LM)

a. Female, entire; b. Male, entire; c, d. Anterior region in median view; e. Female, genital system; f. Female, caudal region; g. Pharyngeal bulb and cardia. h. Lip region, surface view;

i. Male, posterior body region; j. Male, caudal region and spicules; $k$. Female, prevulval uterine sac and vagina (Scale bars: $\mathrm{a}, \mathrm{b}=200 \mu \mathrm{m} ; \mathrm{c}, \mathrm{d}, \mathrm{h}=5 \mu \mathrm{m} ; \mathrm{e}, \mathrm{i}=20 \mu \mathrm{m} ; \mathrm{f}, \mathrm{g}, \mathrm{j}, \mathrm{k}=10 \mu \mathrm{m}$ ).

\section{REFERENCES}

1. Baqri Q.H., 1991. Contribution to the fauna of Sikkim. Nematodes associated with citrus from Sikkim, India. Records of the
Zoological Survey of India, Occasional Paper 128, 103 pp.

2. Choi Y. E. \& Jairajpuri M. S., 1998. Systematic study of Dorylaimida from 
Korea. Three new and four known species of Dorylaimida from Korea. Journal AsiaPacific Entomology, 1(2): 191-209.

3. Dhanam M. \& Jairajpuri M. S., 1999. New leptonchid nematodes: One new genus and eleven new species from Malnad Tracts of Karnataka, India. International Journal of Nematology, 9(1): 1-18.

4. Nguyen T.A.D., Vu T.T.T., Ahmad W. \& Peña-Santiago R., 2011. New observations on Thornedia opisthodelphis (Jairajpuri, 1968) Siddiqi, 1982 (Dorylaimida, Leptonchidae) from Vietnam, with discussion on the identity of the genus. Journal of Nematode Morphology and Systematics, 14:63-69.

5. Goodey T., 1963. Soil and fresh-water nematodes. London, Methuen. $2^{\text {nd }}$ ed. Revised by J. B Goodey, 544pp.

6. Goseco C.G., Ferris V. R. \& Ferris J.M., 1974. Revision in Leptonchoidae (Nematoda: Dorylaimida). Leptonchus, Proleptonchus, Funaria and Meylis n. gen. in Leptonchidae, Leptonchinae. Research bulletin. Agricultural Experiment Station, West Lafayette, Indiana No. 911, 32pp.

7. Heyns J., 1963. Five new species of Leptonchidae (Nemata: Dorylaimoidea) from South Africa. Proceedings of the Helminthological Society of Washington, 30: 7-15.

8. Jairajpuri M.S. \& Siddiqi A.H., 1964. On a new nematode genus Nordia
(Dorylaimoidea: Nordiinae n. subfam.) with remarks on the genus Longidorella Thorne, 1939. Proceedings of the helminthological Society of Washington, 31: 1-9.

9. Jana A. \& Baqri Q. H., 1981. Nematodes from west Bengal (India). Studies on the species of the superfamily Leptonchoidea (Dorylaimida). Journal Zoology Society India, 33: 1-24.

10. Loof P.A.A., 1964. Free-living and plantparasitic nematodes from Venezuela. Nematologica, 10: 201-300.

11. Lordello L.G.E., 1955. On the morphology of Proleptonchus aestivus n. gen., n. sp. and Dorylaimus lourdesae n. sp., two new soil nematodes from Brazil. Proceedings of the Helminthological Society of Washington, 22: 71-75.

12. Siddiqi M.R., 1964. On the occurrence of Enchodorella okhlaensis Jairajpuri \& Siddiqi, 1964 in Jhelum city West Pakistan, with notes on the synonymy of Nodia Jairapuri \& Siddiqi, 1964 (Nematoda: Dorylaimida). Labdev Journal of Science and Technology: 2-208.

13. Thorne G., 1939. A monograph of the nematodes of the superfamily Dorylaimoidea. Capita Zoologica, 8: 1-261.

14. Timm R. W., 1964. Nematodes of the superfamily Dorylaimoidea from East Pakistan. Proceedings of the Helminthological Society of Washington, 31: 144-153.

\title{
PHÁT HIÊN MỚI HAI LOÀI TUYẾN TRÙNG THUỘC HỌ LEPTONCHIDAE (NEMATODA: DORYLAIMIDA) CHO KHU HẸ VIẸT NAM Ở VƯờN QUỐC GIA CÚC PHƯƠNG
}

\author{
Nguyễn Thị Ánh Dương ${ }^{1}$, Vũ Thị Thanh Tâm ${ }^{1}$, Reyes Peña-Santiago ${ }^{2}$ \\ ${ }^{(1)}$ Viện Sinh thái và Tài nguyên sinh vật, Viện Khoa học và Công nghệ Việt Nam \\ ${ }^{(2)} Đ a ̣ i$ học tổng hợp Jaén, Tây Ban Nha
}

\section{TÓM TẮT}

Tuyến trùng sống tự do trong đất họ Leptonchidae là một trong những họ thuộc bộ Dorylaimida ít được biết đến nhất ở Việt Nam, cho đến nay mới chỉ có 1 loài Thornedia opisthodelphic Jairajpuri, 1968 được phát 
hiện từ vườn quốc gia Cúc Phương. Bài báo này ghi nhận thêm 2 loài tuyến trùng cho khu hệ Việt Nam là Proleptonchus aestivus Lordello, 1955 và Tyleptus projectus Thorne, 1939.

Loài Proleptonchus aestivus Lordello, 1955 có kích thước cơ thể L = 1,14-1,21 mm; vùng môi nhô cao hình mũ với các môi hợp lại với nhau. Odontostyle rất mảnh mai với lumen rõ ràng, có chiều dài gấp 10-12 lần chiều rộng. Thực quản có 2 phần tách biệt khá rõ ràng. Con cái có hệ sinh sản đơn kiểu mono-prodelphic với nhánh phía trước phát triển, nhánh phía sau tiêu giảm thành túi tử cung với chiều dài bằng 1,6 lần chiều rộng của cơ thể tại vulva. Đuôi ngắn, mút đuôi tù tròn.

Loài Tyleptus projectus Thorne, 1939 có kích thước cơ thể trung bình, $\mathrm{L}=0,8-0,95 \mathrm{~mm}$, vùng môi hơi tách biệt với đường viền cơ thể và có 6 phần phụ nhô cao quanh miệng. Odontostyle ngắn, lumen rõ ràng. Thực quản có 2 phần tách biệt khá rõ ràng với phần phía sau hình quả lê, có chiều dài tương đương với chiều rộng của cơ thể tại vị trí thực quản. Con cái có hệ sinh sản đơn kiểu mono-opisthodelphic với nhánh phía sau phát triển. Con đực có 2 nhánh sinh sản phát triển đều nhau. Đuôi ngắn với mút đuôi tù tròn.

Tù khóa: Dorylaimida, Leptonchidae, ghi nhận mới, tuyến trùng, Cúc Phương, Việt Nam.

Ngày nhận bài: 10-7-2012 